ELIEL DE SOUZA ${ }^{1}$

Leonardo Ferreira Nobre de SouzA ${ }^{2}$ MILENA DA COSTA BATISTA ${ }^{2}$

Juleta Alice Morena Rodrigues ${ }^{2}$

Isabelle Braz de Oliveira Silva ${ }^{2}$

Hé lo Humberto Angotti Carrara ${ }^{3}$

Técia Maria de Oliverra Maranhã̃o ${ }^{4}$

\section{Biópsia mamária realizada pela técnica de biópsia helicoide: estudo experimental}

\author{
Breast biopsy performed by the belicoid biopsy technique: an \\ experimental study
}

Artigo original

Palavras-chave

Neoplasias da mama/patologia Neoplasias da mama/diagnóstico Neoplasias da mama/cirurgia Biópsia por agulha/métodos

Keywords

Breast neoplasms/pathology Breast neoplasms/diagnosis Brreast neoplasm's/surgery Needle, biopsy/methods

\section{Resumo}

OBJETIVO: avaliar o desempenho da biópsia helicoide na realização de biópsias mamárias. MÉTODOS: foi selecionado aleatoriamente uma amostra composta de 30 pacientes portadoras de câncer de mama submetidas à mastectomia. Foram excluídas as mulheres portadoras de tumor que tivessem consistência pétrea, não-palpável, com manipulação cirúrgica prévia ou que contivesse líquido. Utilizando-se o kit de biópsia helicoide e um equipamento de core biopsy com cânula e agulha de 14 gauge, respectivamente, coletou-se um fragmento por equipamento em área sã e nos tumores, em cada peça cirúrgica, totalizando 120 fragmentos para estudo histológico. Para a análise dos dados, definiu-se um nível de confiança de 95\% e utilizou-se o software SPSS, versão 13; o índice de concordância Kappa e o teste paramétrico t de Student. RESULTADOS: a média das idades das pacientes foi de 51,6 anos ( \pm 1 1, 1 anos). A core biopsy apresentou sensibilidade de 93,3\%, especificidade de 100\% e acurácia de 96,7\%, enquanto a biópsia helicoide teve sensibilidade de 96,7\%, especificidade de 100\% e acurácia de 98,3\%. Na comparação entre a histologia dos tumores e dos fragmentos de biópsias, houve alto grau de concordância nos diagnósticos (Kappa igual a 0,9 , com p<0,05). CONCLUSÕES: ambos os equipamentos proporcionaram o diagnóstico histológico das lesões com alta acurácia. Os resultados deste estudo demonstraram que a biópsia helicoide é uma alternativa confiável no diagnostico pré-operatório de lesões mamárias.

\section{Abstract}

PURPOSE: to assess the helicoid biopsy performance when carrying out breast biopsies. METHODS: thirty patients with breast cancer submitted to mastectomy were selected at random. Women with a tumor of petreous consistency, nonpalpable, submitted to previous surgical manipulation or containing fluid were excluded. The helicoid biopsy kit and a core biopsy device with a cannula and a 14-gauge-needle, respectively, were used to collect a fragment each from a healthy area and from the tumor of each surgical specimen, for a total of 120 fragments for histological study. Data were analyzed statistically by the parametric Student's t-test and by the Kappa concordance index at the $95 \%$ confidence level, using the SPSS software, version 13. RESULTS: the mean patient's age was $51.6( \pm 11.1)$ years old. The core biopsy showed 93.3\% sensitivity, 100\% specificity and 96.7\% accuracy, and the helicoid biopsy showed $96.7 \%$ sensitivity, $100 \%$ specificity, and $98.3 \%$ accuracy. The comparison of tumor histology and biopsy fragments revealed a high degree of concordance in the diagnoses (Kappa equal to 0.9, with $p<0.05$ ). CONCLUSIONS: both methods provided a highly accurate histological diagnosis of the lesions. The results of the present study demonstrate that the helicoid biopsy is a reliable alternative for the preoperative diagnosis of breast lesions.
Ambulatório de Mastologia do Hospital Universitário Onofre Lopes da Universidade Federal do Rio Grande do Norte - UFRN Natal (RN), Brasil; Ambulatório de Mastologia da Liga Norte-Riograndense Contra o Câncer - Natal (RN), Brasil; Programa de Pós-graduação em Ciências da Saúde da Universidade Federal do Rio Grande do Norte - UFRN - Natal (RN), Brasil.

'Pós-graduando (Mestrado) do Programa de Pós-Graduação em Ciências da Saúde do Centro de Ciências da Saúde da Universidade Federal do Rio Grande do Norte - UFRN - Natal (RN), Brasil.

2Bolsista de Iniciação Científica; Aluno de Graduação do Curso de Medicina da Universidade Federal do Rio Grande do Norte UFRN - Natal (RN), Brasil.

${ }_{3}^{3}$ Professor Doutor do Departamento de Ginecologia e Obstetrícia da Faculdade de Medicina de Ribeirão Preto da Universidade de São Paulo - USP - Ribeirão Preto (SP), Brasil.

${ }_{4}^{4}$ Coordenadora do Programa de Pós-graduação do Centro de Ciências da Saúde da Universidade Federal do Rio Grande do Norte - UFRN - Natal (RN), Brasil.

Conflito de interesse: não há. 


\section{Introdução}

$\mathrm{Na}$ avaliação inicial dos nódulos mamários sólidos palpáveis ou nas lesões não-palpáveis com algum grau de suspeita, é necessário realizar uma biópsia para estudo histopatológico. Havendo diagnóstico de câncer, deve-se acrescentar o estudo imuno-histoquímico para determinação da expressão da proteína receptora tipo 2 do fator de crescimento epidérmico humano (HER2), e avaliar a expressão dos receptores de estrógeno (RE) e de progesterona (RP) nas amostras ${ }^{1}$, principalmente se houver indicação de tratamento neoadjuvante.

Os principais equipamentos existentes para biópsia mamária possuem sistemas automáticos ou semiautomáticos, com agulha grossa ou cânula rotativa para colher as amostras ${ }^{2,3}$. No Brasil, o alto custo dessas novas tecnologias parece limitar a sua utilização em serviços de Mastologia ${ }^{4}$. Em vários países, a core biopsy (CB) com agulha de $14 \mathrm{G}$ é preferível para a realização dessas biópsiass, ${ }^{5,6-15}$, apesar de apresentar algumas limitações como: menor eficácia para predizer invasão ${ }^{16}$, não definir o diagnóstico de lesões compostas de tipos histológicos diferentes associados ${ }^{17,18}$ e ter a eficácia reduzida na coleta de fragmentos de nódulos menores que $10 \mathrm{~mm}$ de diâmetro ${ }^{19-22}$.

Considerando a necessidade de se aumentar a quantidade de tecido coletado nas amostras para garantir a alta acurácia das biópsias, neste estudo experimental, avaliou-se comparativamente o desempenho de uma nova técnica de biópsia mamária ${ }^{23}$.

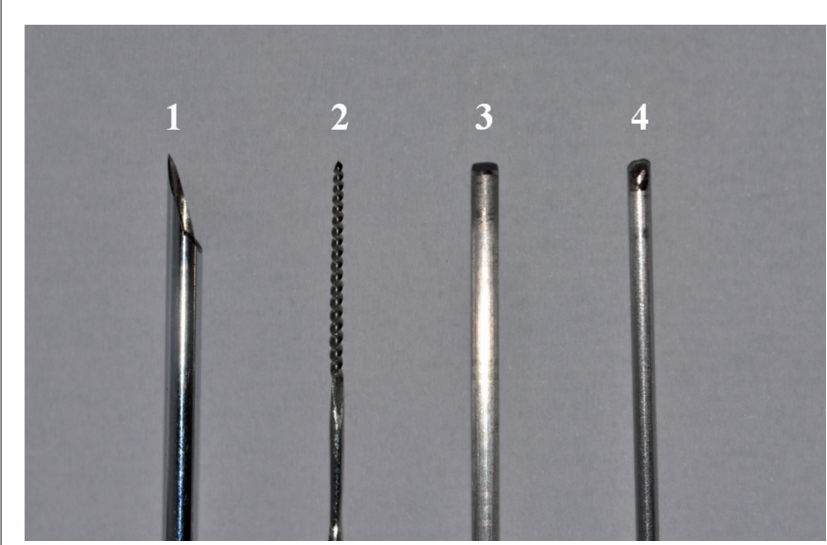

Os componentes estão dispostos pela ordem de utilização. 1 - cânula de $11 \mathrm{G}$ medindo $12,5 \mathrm{~cm}$ de comprimento com ponta bizelada, utilizada para guiar a cânula coletora durante a realização da biópsia; 2 - haste helicoide com $15 \mathrm{~cm}$ de comprimento e espessura de $16 \mathrm{G}$, tendo a ponta helicoide (em forma de parafuso) com área ativa (rosqueada) medindo 2,5 cm de comprimento para fixar o fragmento; 3 - cânula coletora de 14 $G$ medindo $16 \mathrm{~cm}$ de comprimento com extremidade coletora cortante não-bizelada, para cortar a amostra; 4 - mandril de $16 \mathrm{G}$ medindo 12,5 $\mathrm{cm}$ de comprimento utilizado para recolher a amostra do interior da cânula coletora.

Figura 1 - Componentes do kit para biópsia helicoidal.

\section{Métodos}

Este estudo experimental foi desenvolvido em conformidade com a resolução 196/96 do Conselho Nacional de Saúde, tendo aprovação do Comitê de Ética em Pesquisa do Hospital Universitário Onofre Lopes da Universidade Federal do Rio Grande do Norte (HUOL-UFRN), sob registro n ${ }^{\circ}$ 095/97 de 2007, com aplicação do termo de consentimento livre e esclarecido.

As 762 pacientes com câncer mamário, atendidas de fevereiro de 2008 a abril de 2010, foram escolhidas de maneira prospectiva e aleatoriamente. Em uma amostra de 30 mulheres com tumor palpável submetidas à mastectomia, foram excluídas as que tinham tumor de consistência pétrea, não-palpável, com manipulação cirúrgica prévia ou composto de líquido. O equipamento de CB utilizado neste estudo constou de uma pistola tipo Pro-Mag 1.2 Manan, com agulha de $14 \mathrm{G}$ que já tem uso definido em Mastologia ${ }^{5,24}$. O kit da biopsia helicoide (BH) consta dos componentes mostrados na Figura 1, que são acoplados a um micromotor elétrico com rotação ajustável de uso odontológico (caneta odontológica), modelo GRD804 Belte 350 x 270 LB100.

Após a mastectomia, no centro cirúrgico, utilizando-se um dos equipamentos de cada vez, colheu-se um fragmento de região sadia da mama e, subsequentemente, outro fragmento do tumor em cada peça cirúrgica, obedecendo-se o seguinte processo: incisão de 3,0 mm com lâmina de bisturi . $^{\circ} 11$ na pele da mama. $\mathrm{NaCB}$, seguiu-se a técnica já estabelecida ${ }^{25}$, na qual uma agulha grossa envolta por uma cânula e acoplada a uma pistola é introduzida na mama. A amostra da lesão é coletada numa chanfradura da ponta da agulha após o disparo de um mecanismo, o qual projeta a ponta da agulha e da cânula para dentro da lesão, resultando no corte do fragmento.

Para realizar a $\mathrm{BH}$, inicialmente, introduz-se a cânula de $11 \mathrm{G}$ (componente um, Figura 1) pela incisão cutânea, em seguida introduz-se a haste helicoide de 16 G, (componente dois, Figura 1) pela luz da cânula de $11 \mathrm{G}$ com movimento rotatório manual para a direita, avançando-a até que ocorra a fixação do fragmento em sua ponta helicoide. Feito isto, passa-se a cânula coletora, (componente três, Figura 1) acoplada ao micromotor desligado, por dentro da cânula de proteção e englobando a haste helicoide.

Ao ligar-se o micromotor, com aproximadamente 200 RPM, promove-se o avanço da cânula coletora sobre o tecido preso à ponta da haste helicoide, cortando-se uma amostra cilíndrica no seu entorno, ficando o conjunto (haste de fixação e fragmento) alojado na luz da cânula coletora. Nos tumores endurecidos, aumenta-se a rotação do micromotor até que a cânula coletora corte o fragmento. Isto aconteceu em dois casos exclusos do 
estudo, pois só a $\mathrm{BH}$ pode colher fragmentos. A seguir, desliga-se o micromotor e recua-se a cânula coletora contendo a haste helicoide e o fragmento, desconectando-a do micromotor. Passa-se o mandril pela luz da cânula coletora, impelindo a haste de fixação e o fragmento fixo à sua ponta, eliminando-o na extremidade cortante da cânula coletora. Depois de ser constatada a boa qualidade do fragmento, recolhe-se a cânula de proteção da mama encerrando-se o procedimento no tempo máximo de dez minutos, caso contrário repete-se o processo.

Cada fragmento colhido foi colocado sobre papel de filtro para mensuração, sendo depois inserido em um frasco com peso padronizado contendo solução de formol neutro a $10 \%$ para fixação. Logo, o frasco foi pesado por três vezes em balança analítica de cinco dígitos aferida pelo Instituto Nacional de Metrologia, Normalização e Qualidade Industrial (INMETRO). A média dos pesos de cada fragmento foi obtida por diferença simples, segundo a fórmula: $\mathrm{P}_{1}-\mathrm{P}_{0}=\mathrm{PF}\left(\mathrm{P}_{0}=\right.$ peso do frasco contendo formol e o papel de filtro, sem o fragmento; P1=peso do frasco contendo formol e o fragmento com o papel de filtro; $\mathrm{PF}=$ peso do fragmento)

As amostras foram codificadas e encaminhadas ao laboratório de patologia em tempo diferente daquele do envio das respectivas peças cirúrgicas, onde receberam processamento habitual, sendo embebidas em parafina, cortadas e coradas pela técnica de hematoxilina-eosina para estudo histológico em microscópio óptico, por um mesmo patologista familiarizado com as doenças mamárias.

$\mathrm{Na}$ análise dos resultados, considerou-se um nível de significância de 0,05 (5\%) e utilizou-se o software estatístico SPSS, versão 13, e o Excel. O teste de concordância de Kappa foi utilizado para fazer comparações entre os resultados dos exames histopatológicos dos tumores em relação aos fragmentos de biópsias $(\mathrm{CB}$ e $\mathrm{BH})$. Por meio do teste paramétrico $t$ de Student, foi avaliada a diferença quanto ao peso e comprimento dos fragmentos das biópsias (BH e Core). A ferramenta estatística boxplot foi utilizada para avaliar a dispersão dos pesos e dos comprimentos dos fragmentos.

\section{Resultados}

As pacientes deste estudo tinham diagnóstico prévio de câncer de mama obtido em fragmentos de biópsia por agulha grossa e classificado pela $\mathrm{UICC}^{26}$. A média das idades das pacientes foi de 51,6 anos, tendo a mais jovem 34 anos e a mais idosa, 77. Seis pacientes (20\%) tinham menos de 40 anos de idade. A mama direita foi a mais acometida, com 20 casos $(66,7 \%)$. A média das medidas dos diâmetros dos tumores foi de $37,9 \mathrm{~mm}$, tendo uma variação de 13 a $120 \mathrm{~mm}$. Uma paciente tinha doença em estádio clínico I; três em estádio clínico IIA (T2NOM0) e uma em estádio clínico (TisNOM0) com tumor palpável. Vinte e cinco pacientes $(83,3 \%)$ tinham doença em estádios clínicos avançados (IIB, IIIA, IIIB, IIIC e IV).

Entre os tumores, o carcinoma ductal infiltrante (CDI) puro foi a neoplasia mais frequente, sendo diagnosticada em 13 casos $(43,3 \%)$. Das lesões com histologia mista, o CDI associado a focos microscópicos esparsos de carcinoma ductal in situ (CDI) foi diagnosticado em dez casos $(33,3 \%)$. Considerando-se isoladamente o componente infiltrante dos tumores, o CDI teve incidência de 26 casos $(86,6 \%)$. Outros tipos de tumores ocorreram em sete casos $(23,3 \%)$.

A comparação entre os dois equipamentos feita com a aplicação do teste $t$ de Student para amostras pareadas demonstrou que, utilizando-se a $\mathrm{BH}$, foram coletados fragmentos significativamente mais pesados e com maior comprimento do que com a CB. Em áreas sãs, os fragmentos da $\mathrm{BH}$ mediram em média $2,2 \mathrm{~mm}$ a mais de comprimento e pesaram em média $14,2 \mathrm{mg}$ a mais, e nos tumores, mediram em média $10,3 \mathrm{~mm}$ a mais de comprimento e pesaram em média 23,1 mg a mais. Nas áreas de tumor, foram obtidos fragmentos mais pesados do que nas áreas sãs, utilizando-se ambos os equipamentos. A distribuição do peso e do comprimento dos fragmentos obtidos pelos equipamentos nas áreas sãs e nos tumores estão demonstrados nas Figuras 2 e 3.

Comparando-se a histologia dos fragmentos da $\mathrm{BH}$ em relação ao exame histopatológico dos tumores, considerando-se isoladamente os componentes infiltrantes e em um caso, o diagnóstico de carcinoma ductal in situ de alto grau com extensão lobular, houve concordância diagnóstica em 29 casos (96,6\%), havendo um resultado falso-negativo $(3,3 \%)$. Em uma comparação similar feita com a CB, houve concordância diagnóstica em 27 casos $(90 \%)$, dois resultados falsos-negativos e um caso de discordância diagnóstica em relação à histologia de um tumor com componente CDI associado ao CDI com

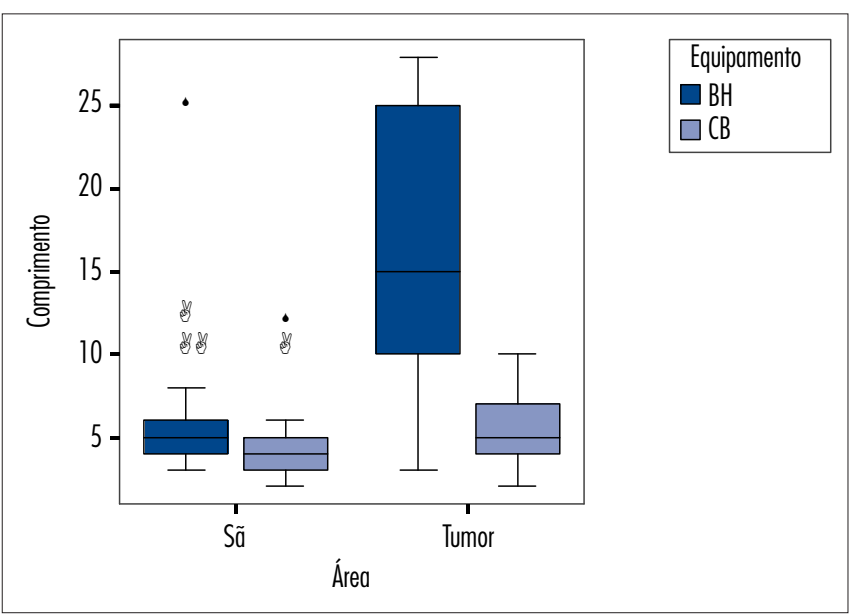

Figura 2 - Box-plot do comprimento (em milímetros) dos fragmentos de acordo com a área e o equipamento utilizado ( $\mathrm{CB}$ e $\mathrm{BH})$. 


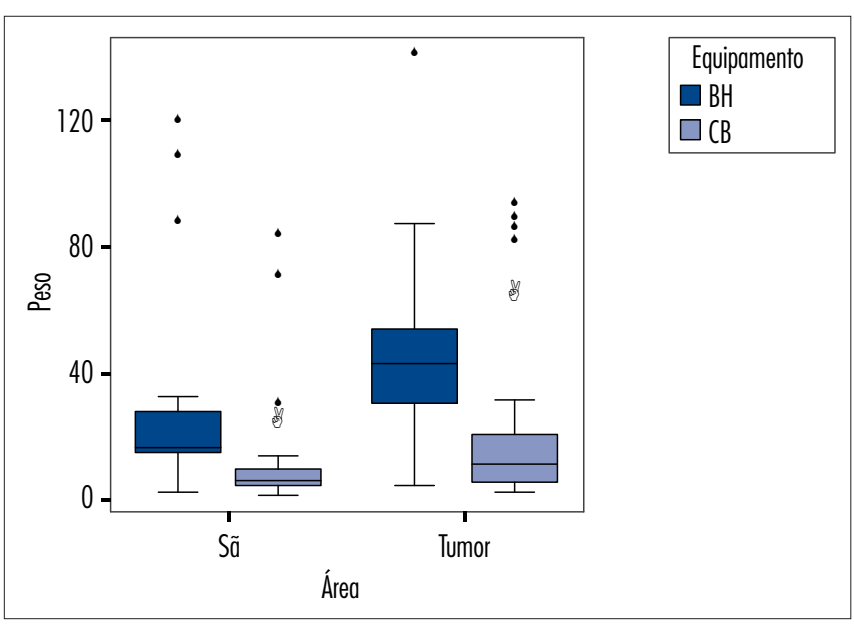

Figura 3 - Box-plot do peso (mg) dos fragmentos de acordo com a área e o equipamento utilizado ( $\mathrm{CB}$ e Kit $\mathrm{BH})$.

carcinoma coloide, no qual o fragmento da CB acusou apenas a presença de componente in situ.

$\mathrm{Na}$ análise comparativa da histologia dos fragmentos da $\mathrm{CB}$ e da BH entre si, houve concordância diagnóstica em 26 casos $(86,6 \%)$. Os quatro resultados discordantes foram dois falso-negativos da core $(6,6 \%)$, um caso falso-negativo da $\mathrm{BH}$ e um caso em que a histologia do fragmento da $\mathrm{CB}$ apresentou diagnóstico de lesão in situ e o fragmento da $\mathrm{BH}$ revelou componentes infiltrantes mistos.

Comparando-se a histologia dos fragmentos da CB em relação aos tumores, ao considerar isoladamente o componente infiltrante, foi constatado que a estatística $\mathrm{K}$ (Kappa) foi de 0,93 com $\mathrm{p}<0,05$, havendo concordância entre os resultados com sensibilidade de 93,3\% e acurácia de $96,7 \%$. Essa mesma análise feita em relação aos fragmentos colhidos pela $\mathrm{BH}$ constatou que a estatística $\mathrm{K}$ foi de 0,97 com $\mathrm{p}<0,05$, a sensibilidade foi de 96,7 e a acurácia de 98,3\% em ambas as situações, considerandose os valores de Kappa segundo Landis e $\mathrm{Koch}^{27}$. Neste estudo, não houve resultados falso-positivos nem amostras consideradas insuficientes para o diagnóstico.

\section{Discussão}

Há várias indicações que permitem atribuir a interferência do volume da amostra de biópsia no resultado do exame histopatológico de lesões mamárias ${ }^{24,28-32}$. Neste estudo, coletou-se apenas um fragmento nas biópsias, constatando-se que este número foi suficiente para definir de forma significativa os diagnósticos. Isto ficou demonstrado principalmente quando se utilizou a $\mathrm{BH}$, obtendo-se fragmentos com maior comprimento e mais pesados, havendo concordância diagnóstica entre a histologia dos fragmentos e dos tumores em 98,3\%. Entretanto, é preciso considerar que ao utilizar ambos os equipamentos (BH e $\mathrm{CB}$ ), houve resultados falso-negativos e discordância diagnóstica, os quais indicam a necessidade de se colher mais de um fragmento em biópsias de lesões mamárias palpáveis.

No exame histológico dos fragmentos coletados por ambos os equipamentos, os diagnósticos obtidos tiveram concordância significativa e, ao contrário do que é citado na literatura a respeito da subestimação diagnóstica de componentes infiltrantes nas biópsias por agulha grossa ${ }^{33-35}$, neste estudo, a subestimação ocorreu em relação aos focos microscópicos de carcinoma in situ peritumorais ou esparsos, nos casos em que o CDI estava associado ao mesmo. Tal ocorrência, entretanto, não parece ter grandes implicações clínicas, visto que a lesão principal era o CDI que foi diagnosticado, e que a subestimação ocorrida não teria implicações na estratégia terapêutica.

Do ponto de vista operacional, a $\mathrm{BH}$ se mostrou um equipamento com boa portabilidade, seguro, confiável e de fácil operacionalização para a coleta de amostras de lesões mamárias em regime ambulatorial.

Conclui-se que a BH mostrou-se eficiente para a realização de biópsia de tumores mamários palpáveis, e que os resultados obtidos justificam o pedido de registro de patente da inovação e o início de um estudo in vivo mais aprofundado com um número amostral maior, incluindo lesões com histologia diversificada e tamanhos diferentes, além das não-palpáveis, para se avaliar a acurácia e os aspectos relacionados ao grau de desconforto e possíveis complicações da utilização da $\mathrm{BH}$, além de outros parâmetros que definam melhor a contribuição do equipamento no diagnóstico pré-operatório das lesões mamárias.

\section{Agradecimentos}

Aos diretores do Hospital Universitário Onofre Lopes, da Liga Norte-Riograndense contra o Câncer e à coordenação do Programa de Pós-graduação do Centro de Ciências da Saúde da Universidade Federal do Rio Grande do Norte.

\section{Participação dos autores}

Eliel de Souza - Pesquisador responsável pelo estudo.

Leonardo Ferreira de Souza Nobre, Milena da Costa Batista; Julieta Alice Morena Rodrigues e Isabelle Braz de Oliveira Silva - Colaboração no levantamento bibliográfico, tradução de textos, coleta de dados e produção de planilhas.

Hélio Humberto Angotti Carrara - Colaboração na revisão, análise e redação do artigo.

Técia Maria de Oliveira Maranhão - Colaboração como orientadora durante todo o desenvolvimento da pesquisa, revisão, redação e análise do artigo. 
1. D'Alfonso T, Liu YF, Monni S, Rosen PP, Shin SJ. Accurately assessing her-2/neu status in needle core biopsies of breast cancer patients in the era of neoadjuvant therapy: emerging questions and considerations addressed. Am J Surg Pathol. 2010;34(4):575-81.

2. Vlastos G, Verkooijen HM. Minimally invasive approaches for diagnosis and treatment of early-stage breast cancer. Oncologist. 2007;12(1):1-10.

3. Kettritz U, Rotter K, Schreer I, Murauer M, Schulz-Wendtland R, Peter D, et al. Stereotactic vacuum-assisted breast biopsy in 2874 patients: a multicenter study. Cancer. 2004;100(2):245-51.

4. Pistolese CA, Ciarrapico AM, Della Gatta F, Perretta T, Cossu E, Bolacchi F, et al. Cost-effectiveness analysis of two vacuum-assisted breast biopsy systems: Mammotome and Vacora. Radiol Med. 2009; $114(5): 743-56$

5. Fuhrman GM, Cederbonn GJ, Bolton JS, King TA, Duncan JL, Champaign JL, et al. Image-guided core-needle breast biopsy is an accurate diagnostic technique to evaluate patients with nonpalpable imaging abnormalities. Ann Surg. 1998;227(6):932-9.

6. Parker SH, Burbank F, Jackman RJ, Aucreman CJ, Cardenosa G, Cink TM, et al. Percutaneous large-core breast biopsy: a multiinstitutional study. Radiology. 1994;193(2):359-64.

7. Dillon MF, Quinn CM, McDermott EW, O'Doherty A, O'Higgins $N$, Hill AD. Diagnostic accuracy of core biopsy for ductal carcinoma in situ and its implications for surgical practice. J Clin Pathol. 2006;59(7):740-3.

8. Bruening W, Fontanarosa J, Tipton K, Treadwell JR, Launders J, Schoelles K. Systematic review: comparative effectiveness of coreneedle and open surgical biopsy to diagnose breast lesions. Ann Intern Med. 2010;152(4):238-46.

9. Youk JH, Kim EK, Kim M, Oh KK. Sonographically guided 14-gauge core needle biopsy of breast masses: a review of 2,420 cases with long-term follow-up. AJR Am J Roentgenol. 2008;190(1):202-7.

10. Andreu FJ, Sáez A, Sentís $M$, Rey M, Fernández $S$, Dinarès $C$, et al. Breast core biopsy reporting categories-An internal validation in a series of 3054 consecutive lesions. Breast. 2007;16(1):94-101.

1 1. Iftimia NV, Mujat M, Ustun T, Ferguson RD, Danthu V, Hammer DX. Spectral-domain low coherence interferometry/optical coherence tomography system for fine needle breast biopsy guidance. Rev Sci Instrum. 2009;80(2):024302.

12. Ng VY, Thomas K, Crist M, Wakely PE Jr, Mayerson J. Fine needle aspiration for clinical triage of extremity soft tissue masses. Clin Orthop Relat Res. 2010;468(4): $1120-8$.

13. Oyama T, Koibuchi Y, McKee G. Core needle biopsy (CNB) as a diagnostic method for breast lesions: comparison with fine needle aspiration cytology (FNA). Breast Cancer. 2004; 1 1 (4):339-42.

14. Rakha EA, Ellis IO. An overview of assessment of prognostic and predictive factors in breast cancer needle core biopsy specimens. J Clin Pathol. 2007;60(12):1300-6.

15. Rakha EA, Lee AH, Reed J, Murphy A, El-Sayed M, Burrell H, et al. Screen-detected malignant breast lesions diagnosed following benign (B2) or normal (B1) needle core biopsy diagnoses. Eur J Cancer. 2010;46(10):1835-40.

16. Tse GM, Tan PH. Diagnosing breast lesions by fine needle aspiration cytology or core biopsy: which is better? Breast Cancer Res Treat. 2010;123(1):1-8.

17. O'Connor A, Wylie EJ, Nuttall LJ. Complications of breast core biopsy. Breast Cancer Res. 2002;4 Suppl 1:54.

18. Uematsu T, Kasami M. The use of positive core wash cytology to estimate potential risk of needle tract seeding of breast cancer: directional vacuum-assisted biopsy versus automated core needle biopsy. Breast Cancer. 2010;17(1):61-7.

19. Martins EC, Soares A, Guimarães CM, Freire MN, Koch H, Marsillac M. O uso da agulha de $16 G$ na core biopsy guiada por ultrassonografia em lesões mamárias. Rev Col Bras Cir. 2009;36(4):312-5

20. Han BK, Choe YH, Ko YH, Nam SJ, Kim JH, Yang JH. Stereotactic core-needle biopsy of non-mass calcifications: outcome and accuracy at long-term follow-up. Korean J Radiol. 2003;4(4):217-23.

21. Liberman L, Dershaw DD, Rosen PP, Abramson AF, Deutch BM, Hann LE. Stereotaxic 14-gauge breast biopsy: how many core biopsy specimens are needed? Radiology. 1994;192(3):793-5.

22. Camargo Júnior HSA, Camargo MMA, Teixeira SRC, Arruda MS. Biópsia de fragmento em nódulos mamários suspeitos com até 10 mm. Rev Bras Ginecol Obstet. 2007;29(6):317-23.

23. Brenner RJ, Fajardo L, Fisher PR, Dershaw DD, Evans WP, Bassett $L$, et al. Percutaneous core biopsy of the breast: effect of operator experience and number of samples on diagnostic accuracy. AJR Am J Roentgenol. 1996; 166(2):341-6.

24. Parker SH, Lovin JD, Jobe WE, Burke BJ, Hopper KD, Yakes WF. Nonpalpable breast lesions: stereotactic automated large-core biopsies. Radiology. 1991;180(2):403-7.

25. Parker SH, Lovin JD, Jobe WE, Luethke JM, Hopper KD, Yakes WF, et al. Stereotactic breast biopsy with a biopsy gun. Radiology. 1990;176(3):741-7.

26. Brasil. Ministério da Saúde. Secretaria de Atenção à Saúde. Instituto Nacional de Câncer [Internet]. TNM: classificação de tumores malignos. 6a ed. Rio de Janeiro: INCA; 2004 [citado 2010 Jul 10]. Disponível em: http://www.inca.gov.br/tratamento/tnm/tnm2.pdf

27. Landis JR, Koch GG. The measurement of observer agreement for categorical data. Biometrics. 1977;33(1):159-74.

28. Abreu-e-Lima MC, Maranhão N, Almeida V, Melo CB, Araújo $E$, Abreu-e-Lima $M$, et al. Comparação entre fragmentos obtidos com agulhas de calibres 14 e 12 em "core biopsy" estereotáxica de lesões mamárias impalpáveis: diferenças entre o tamanho dos fragmentos e frequência dos tipos de lesões diagnosticadas. Radiol Bras. 2001;34(5):255-60.

29. Joshi A, Maimoon S. Limitations of fine needle aspiration cytology in subtyping breast malignancies - a report of three cases. J Cytol. 2007;24(4):203-6.

30. Parker SH, Klaus AJ. Performing a breast biopsy with a directional, vacuumassisted biopsy instrument. Radiographics. 1997;17(5):1233-52.

31. Crippa CG, D'Avila CLP, Schaefer MB, Oliveira, AR, Traebert EE. A mamotomia no diagnóstico e terapêutica de lesões mamárias. Femina. 2006;34(10):687-93.

32. Liberman L, Dershaw DD, Rosen PP, Giess CS, Cohen MA, Abramson $\mathrm{AF}$, et al. Stereotaxic core biopsy of breast carcinoma: accuracy at predicting invasion. Radiology. 1995;194(2):379-81.

33. Houssami N, Ciatto S, Ellis I, Ambrogetti D. Underestimation of malignancy of breast core-needle biopsy: concepts and precise overall and category-specific estimates. Cancer. 2007;109(3):487-95.

34. Ciatto S, Houssami N, Ambrogetti D, Bianchi S, Bonardi R, Brancato $B$, et al. Accuracy and underestimation of malignancy of breast core needle biopsy: the Florence experience of over 4000 consecutive biopsies. Breast Cancer Res Treat. 2007;101(3):291-7.

35. Sullivan ME, Khan SA, Sullu Y, Schiller C, Susnik B. Lobular carcinoma in situ variants in breast cores: potential for misdiagnosis, upgrade rates at surgical excision, and practical implications. Arch Pathol Lab Med. 2010;134(7):1024-8. 